\title{
Prolonged Microgravity Affects Human Brain Structure and Function
}

\author{
(DD.R. Roberts, DD. Asemani, (DP.J. Nietert, (DM.A. Eckert, (DD.C. Inglesby, (DJ.J. Bloomberg, (DM.S. George, and (DT.R. Brown
} if $\mathrm{O} \equiv$

\section{ABSTRACT}

BACKGROUND AND PURPOSE: Widespread brain structural changes are seen following extended spaceflight missions. The purpose of this study was to investigate whether these structural changes are associated with alterations in motor or cognitive function.

MATERIALS AND METHODS: Brain MR imaging scans of National Aeronautics and Space Administration astronauts were retrospectively analyzed to quantify pre- to postflight changes in brain structure. Local structural changes were assessed using the Jacobian determinant. Structural changes were compared with clinical findings and cognitive and motor function.

RESULTS: Long-duration spaceflights aboard the International Space Station, but not short-duration Space Shuttle flights, resulted in a significant increase in total ventricular volume (10.7\% versus $0 \%, P<.001, n=12$ versus $n=7)$. Total ventricular volume change was significantly associated with mission duration $(r=0.72, P=.001, n=19)$ but negatively associated with age $(r=-0.48, P=.048$, $n=19$ ). Long-duration spaceflights resulted in significant crowding of brain parenchyma at the vertex. Pre- to postflight structural changes of the left caudate correlated significantly with poor postural control; and the right primary motor area/midcingulate correlated significantly with a complex motor task completion time. Change in volume of 3 white matter regions significantly correlated with altered reaction times on a cognitive performance task (bilateral optic radiations, splenium of the corpus callosum). In a post hoc finding, astronauts who developed spaceflight-associated neuro-ocular syndrome demonstrated smaller changes in total ventricular volume than those who did not $(12.8 \%$ versus $6.5 \%, n=8$ versus $n=4)$.

CONCLUSIONS: While cautious interpretation is appropriate given the small sample size and number of comparisons, these findings suggest that brain structural changes are associated with changes in cognitive and motor test scores and with the development of spaceflight-associated neuro-optic syndrome.

ABBREVIATIONS: $C D S=$ code substitution; $C P T=$ continuous performance; $\% \Delta V V=$ percentage total ventricular volume change; FTT Functional Task Test; $\quad$ ISS = International Space Station; LSAH = Lifetime Surveillance of Astronaut Health; NASA = National Aeronautics and Space Administration; PVE $=$ partial volume estimation; SANS = spaceflight-associated neuro-ocular syndrome; WinSCAT = Spaceflight Cognitive Assessment Tool for Windows

7 he effects of spaceflight on the human brain must be understood to ensure the safety of astronauts who participate in long-duration missions aboard the International Space Station (ISS) and to support future space-exploration missions. We have previously shown an upward shift of the brain, crowding of

Received June 2, 2019; accepted after revision August 19.

From the Departments of Radiology and Radiological Science (D.R.R., D.A., D.I. T.R.B.), Department of Neurosciences (D.R.R.), Public Health Sciences (P.J.N.), Otolaryngology-Head and Neck Surgery (M.A.E.), and Psychiatry and Behavioral Sciences (M.S.G.), Medical University of South Carolina, Charleston, South Carolina; Neurosciences Laboratory (J.J.B.), NASA Johnson Space Center, Houston, Texas; and Ralph H. Johnson VA Medical Center (M.S.G.), Charleston, South Carolina.

This study was funded by a grant from the National Aeronautics and Space Administration (NNX13AJ92G). Dr Nietert's time on this project was funded, in part, by the National Institutes of Health National Center for Advancing Translational Science (grant No. UL1-TR001450) and the National Institute of General Medical Sciences (grant No. U54-GM104941). Dr Inglesby's time on this project was funded by a Minorities in STEM Student Scholarship from the South Carolina Space Grant Consortium (grant No. NNX15AL49H). eloquent brain tissue at the vertex, and enlargement of the ventricular system in astronauts following long-duration missions aboard the ISS. ${ }^{1}$ Similarly, other investigators have described local

All study data were provided by the NASA Lifetime Surveillance of Astronaut Health office. The study was approved by the NASA Johnson Spaceflight Center and the Medical University of South Carolina institutional review boards. All participants provided written informed consent for use and publication of their data. NASA has reviewed the manuscript and figures, which preserve astronaut anonymity and are compliant with NASA Astronaut Office privacy standards. To obtain access to the data, one should make application to the NASA Lifetime Surveillance of Astronaut Health Office. Any release of data must be approved by NASA.

Dr. Asemani made significant contributions to this paper as co-first author.

Please address correspondence to Donna R. Roberts, MD, Departments of Radiology and Radiological Science and Neurosciences, Medical University of South Carolina, 96 Jonathan Lucas Street, MSC 323, CSB Suite 210, Charleston, SC 29425; e-mail: robertdr@musc.edu; @BrainsOnMars

-- Indicates open access to non-subscribers at www.ajnr.org

$\equiv$ Indicates article with supplemental on-line appendix and tables.

http://dx.doi.org/10.3174/ajnr.A6249 
nonlinear deformations in astronaut brain structure postflight. ${ }^{2-4}$ While clinically relevant functional decrements in essentially every other body system have been documented following spaceflight, ${ }^{5}$ the etiology and functional significance of these brain structural changes remain unclear. Here, we sought to determine the extent to which pre- to postflight structural brain changes may be associated with changes in astronauts' cognitive or motor performances postflight.

A subset of astronauts develop a constellation of clinical findings, including altered visual acuity, ophthalmologic changes such as cotton wool spots, choroidal folds, and optic disc edema. ${ }^{6}$ The National Aeronautics and Space Administration (NASA) has termed this constellation of clinical findings "spaceflight-associated neuro-ocular syndrome" (SANS). ${ }^{7}$ Therefore, we also examined post hoc the extent to which pre- to postflight structural brain changes were associated with the development of SANS.

We hypothesized that regional brain changes of astronauts identified on postflight MR imaging are progressive on the basis of mission duration, may affect cognitive and motor performance, and may provide insight into the pathophysiology of SANS.

\section{MATERIALS AND METHODS Experimental Design}

The study was approved by the Institutional Review Boards of the NASA Johnson Spaceflight Center and the Medical University of South Carolina. After the nature and possible consequences of the study, including potential loss of privacy, were explained to the astronauts, informed consent was obtained.

For this study, we retrospectively analyzed data obtained from the NASA Lifetime Surveillance of Astronaut Health (LSAH) Program. Due to the retrospective nature of the study, data for some of the tests were not available for all astronauts in the study. This article was reviewed by NASA to ensure astronaut anonymity.

\section{Participants}

The participants in this study represent a subset of astronauts from a previously described NASA astronaut cohort. ${ }^{1}$ The inclusion criterion was any astronaut to date at the time of the study who had undergone both pre- and postflight brain MR imaging. The exclusion criterion was the lack of 3D high-resolution imaging on pre- or postflight brain MR imaging, which was required to perform the quantitative analysis. Of a cohort of 34 astronauts, the necessary pre- and postflight high-resolution imaging was available for 19 astronauts: 7 astronauts (1 woman; mean age, $46.7 \pm 2.1$ years) who underwent short-duration flights aboard the Space Shuttle (14.7 \pm 1.6 days) and 12 astronauts ( 2 women; mean age, $47.5 \pm 4.8$ years) who underwent long-duration missions aboard the ISS $(162.7 \pm 21.8$ days $)$.

\section{Brain MR Imaging Analysis}

For each astronaut, 3D high-resolution MR imaging was performed on a $3 \mathrm{~T}$ Verio system (Siemens, Erlangen, Germany) before and within 3 weeks following spaceflight using the following parameters: T1-weighted magnetization-prepared rapid acquistion of gradient-echo sequence; 176 sagittal slices; TR =
$2300 \mathrm{~ms} ; \mathrm{TE}=2.98 \mathrm{~ms} ; \mathrm{TI}=90 \mathrm{~ms} ;$ flip angle $=7^{\circ} ; \mathrm{FOV}=240 \times$ $256 \mathrm{~mm}^{2}$; matrix $=240 \times 256 ; 1 \times 0.9 \times 0.9 \mathrm{~mm}^{3}$ voxels. Details of the MR imaging analysis are provided in the On-line Appendix. Briefly, the images of the outer skull were used to coregister the pre- and postflight images to correct for head positioning within the MR imaging scanner. Volumetric analysis was performed using publicly available software (FSL, Version 5.0; http://www.fmrib.ox.ac.uk/fsl). Local crowding or displacement of brain tissue along the brain-CSF interface was assessed by subtracting the affine-transformed preflight brain parenchyma image from the skull-aligned postflight brain parenchyma image. Finally, regional deformation of the brain parenchyma was assessed on the basis of the extent of nonlinear warping of the preflight whole brain to the postflight whole brain, as defined by the Jacobian determinant.

\section{Cognitive Performance: Changes in the Spaceflight Cognitive Assessment Tool for Windows Scores}

Cognitive testing was available for the 12 long-duration astronauts who participated in ISS missions, which we obtained from the LSAH. Per NASA protocol, all astronauts on ISS missions participate in the Spaceflight Cognitive Assessment Tool for Windows (WinSCAT) battery, which was developed by NASA as a sensitive screening tool for astronauts to monitor their neurocognitive status while in space and to alert NASA flight surgeons to any alterations in astronaut performance. ${ }^{8,9}$ Because WinSCAT testing was not NASA protocol for Shuttle flights, no data were available for Shuttle astronauts. The WinSCAT is composed of 5 subtests that characterize visual search, processing speed/efficiency, and learning (Code Substitution, CDS); memory (Code Substitution Delayed Recognition, CCD); working memory (Delayed Matching to Sample, MSP); arithmetic (Mathematical Processing, MTH); and sustained attention (Continuous Performance, CPT). Accuracy and reaction time were obtained for each subtest. ${ }^{8,9}$ The WinSCAT has been validated in various patient populations and has proved useful in identifying alterations in cognitive performance with high sensitivity and specificity. ${ }^{8}$ The standard NASA procedure is for ISS astronauts to take the WinSCAT 6 times before flight to allow familiarization with testing. Per the NASA standard, the performances on the last 3 WinSCAT tests taken before flight were averaged and considered baseline for each astronaut. ${ }^{9}$ During the mission onboard the ISS, astronauts took the WinSCAT every 30 days; therefore, depending on the mission length, the number of inflight tests varied among astronauts. Finally, approximately 1 month following return to Earth (mean, $35.2 \pm 4.8$ days), astronauts underwent a postflight WinSCAT. We chose the postflight examination as the comparator study to be consistent with the MR imaging data. The percentage change in WinSCAT scores was calculated as

$$
\% \text { Change Postflight to Preflight }=\frac{\text { Score }_{\text {Post }}-\text { Score }_{\text {Pre }}}{\text { Score }_{\text {Pre }}} \times 100,
$$

where Score $_{\text {Pre }}$ and Score ${ }_{\text {Post }}$ represent the pre- and postflight accuracy and reaction time scores for each of the WinSCAT subtests. 


\section{Motor Performance: Changes in Functional Task Performance and Postural Equilibrium Control}

Eight astronauts (7 long-duration and 1 short-duration) in this study had participated in a separate Functional Task Test (FTT) study. The FTT is a separate NASA-sponsored research study designed to assess the impact of spaceflight on the astronauts' performance of mission-critical tasks required after landing on a planetary surface, for example, hatch opening or egress from a space capsule. ${ }^{10}$ The study protocol had 11 subtests relevant to motor control: Activity Board, Dynamic Postural Stability, Recovery from Fall/Stand, Supine Egress and Walk, Pegboard Test, Seated Egress and Walk, Object Translation, Ladder Climb, Hatch Opening, Jump Down, and Tandem Walk. ${ }^{10}$ More complete descriptions of each subtest are provided elsewhere ${ }^{10}$ and in the On-line Appendix. We obtained the FTT data from the LSAH.

Each ISS astronaut underwent FTT approximately 100 and 60 days before launch and within 24-48 days following return from spaceflight. The average score from the 2 testing sessions before spaceflight was used as the preflight baseline. We calculated the percentage change in scores for each test as

$$
\text { \% Change Postflight to Preflight }=\frac{\text { Score }_{\text {Post }}-\text { Score }_{\text {Pre }}}{\text { Score }_{\text {Pre }}} \times 100,
$$

where Score $_{\text {Pre }}$ and Score $_{\text {Post }}$ represent the pre- and postflight scores for each of the 11 FTT subtests.

\section{Clinical Findings of SANS}

The current diagnostic criteria for SANS have been defined by NASA to include ocular disc edema of variable Frisén grades, globe flattening, choroidal folds, hyperopic refractive error shifts, and cotton wool spots. ${ }^{11}$ Using this definition of SANS, we obtained the ophthalmologic records for each ISS astronaut from the LSAH and screened for any documented clinical signs of SANS.

\section{Statistical Analyses}

Several analytic approaches were used to assess changes in brain MR imaging metrics, and cognitive and motor function scores, and to quantify correlations among relevant study variables. All analyses were conducted using SAS, Version 9.4 (SAS Institute, Cary, North Carolina). Given the relatively small number of cases composing this sample, nonparametric statistical approaches were used throughout. Within-group, pre- to postflight changes in brain MR imaging metrics (ie, volume metrics) were calculated using Wilcoxon signed rank tests, while between-group differences of these changes were calculated using Wilcoxon rank sum tests. Unadjusted and adjusted (partial) Spearman rank-based correlations were calculated using data from all 19 astronauts to quantify the associations between changes in brain MR imaging metrics with age at launch and flight duration.

To estimate local crowding or displacement of brain tissue along the brain-CSF interface within the Shuttle and ISS astronaut groups from the Jacobian modulated pre- to postflight difference image, we used the FSL Randomise function (https://fsl. fmrib.ox.ac.uk/fsl/fslwiki/Randomise) to correct for multiple comparisons across the whole brain, assuming an overall threshold of $P<.05$ based on 5000 random permutations and threshold-free cluster enhancement. ${ }^{12}$

To estimate voxelwise associations between brain volume change and each of the cognitive and motor test scores separately, we obtained nonparametric correlations between the normalized Jacobian determinant and each cognitive and motor test score as a covariate. Again, the FSL Randomise function was used to correct for multiple comparisons across the whole brain. ${ }^{12}$

Spearman correlations were also used to quantify associations between changes in the 12 ISS astronauts' WinSCAT scores, flight duration, and changes in brain MR imaging metrics. Spearman correlations were also used to quantify associations between changes in the 8 astronauts' FTT scores and changes in brain MR imaging metrics. A correlation of $>0.5$ was considered large on the basis of Cohen, 1992. ${ }^{13}$

To correct for multiple comparisons, we used an adaptive linear step-up procedure as suggested by Benjamini et al. ${ }^{14}$ Using this method, we corrected the $P$ value for 21 tests (5 WinSCAT subtests, each with accuracy and reaction time, and 11 FTT subtests) to control the false discovery rate at $5 \%$. The false discovery rate is the expected percentage of "discoveries" (ie, significant findings) that are, in fact, spurious. Instead of the nominal $P$ value $=.05$, we used smaller $P$ value thresholds for flagging significant results. For the comparison between pre- and postflight test scores, a corrected $P$ value of .0476 was used. For the correlations between the percentage total ventricular volume change $(\% \Delta \mathrm{VV})$ and percentage changes in cognitive/motor testing, a corrected $P$ value of .00238 was used.

In a post hoc analysis, we used a Wilcoxon rank sum test to compare $\% \Delta \mathrm{VV}$ among astronauts who developed SANS versus those who did not; because this particular comparison was deemed secondary, the resulting $P$ value was not accounted for when adjusting for multiple comparisons.

\section{RESULTS}

\section{Volumetric Analysis by Brain Tissue Segment}

No significant change was seen pre- to postflight in the total volume of gray matter or white matter for either the Shuttle or ISS astronauts (On-line Table 1). In accordance with our prior study, ${ }^{1}$ there was a significant increase in the total ventricular volume postflight compared with preflight in the ISS astronauts but not in the Shuttle astronauts $(+10.7 \%$ versus $0 \%$, respectively, $P<.001$, Fig $1 A$ ). For the ISS astronauts, the percentage change in ventricular volume for the left lateral, right lateral, third, and fourth ventricles was respectively $17.1 \% \pm$ 7.3\% ( $P=.005), \quad 15.2 \% \pm 8.4 \%(P=.005), 15.4 \% \pm 4.9 \%$ $(P=.005)$, and $-0.83 \% \pm 4.57 \%(P=.68)$. The $\% \Delta V V$ pre- to postflight was significantly associated with the flight duration across astronauts $(r=0.72, P=.001, n=19$, Fig $1 B)$, which remained significant after adjusting for astronauts' ages at time of launch. There was a significant negative association between astronauts' ages and $\% \Delta \mathrm{VV}$ when adjusted for flight duration ( $r=-0.48, P=.048, n=19$, Fig $1 C$ ), with the younger astronauts experiencing larger $\% \Delta \mathrm{VV}$. 

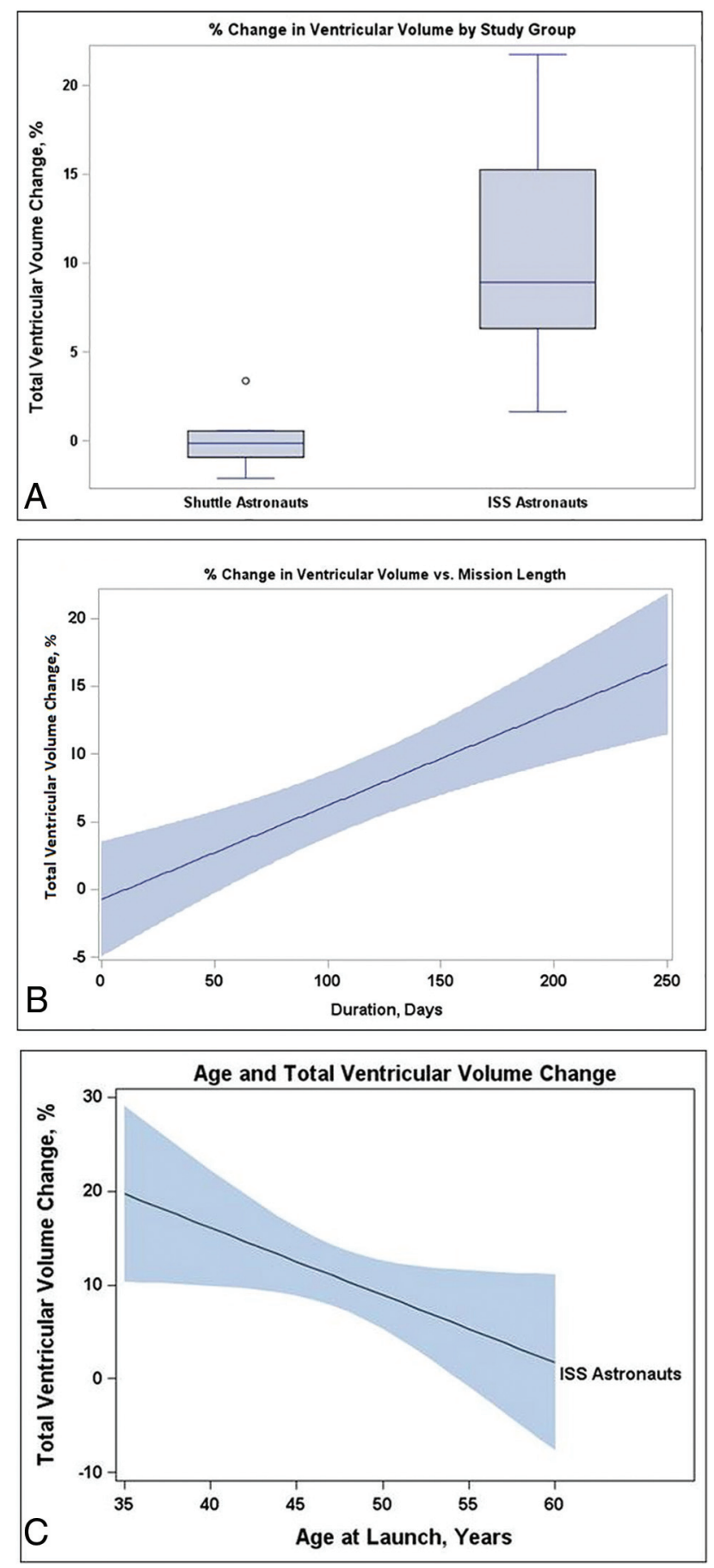

FIG 1. Ventricular volume increases depended on duration in flight, type of space flight, and astronaut age $(A-C)$. Note that the following modifications were made to the figures to protect astronaut anonymity: Individual data points for mission duration and ISS astronaut ages are not plotted. Instead, the regression line and 95\% confidence bands are shown. $A$, Boxplot showing $\% \Delta \mathrm{VV}$ versus mission duration. $B, \% \Delta \mathrm{VV}$ versus mission duration, including Shuttle and ISS astronauts. $C, \% \Delta \mathrm{VV}$ versus ISS astronaut age at launch.

\section{Local Brain Boundary Change Estimation}

After accounting for global shift of the brain, we quantified local crowding or displacement of brain tissue along the brainCSF interface (the boundary of the brain). In the astronauts who had undergone long-duration spaceflights, significant local changes in brain structure were noted (Fig 2). There was significant crowding of brain parenchyma at the vertex involving supplementary motor, premotor, and primary sensorimotor regions, consistent with our previously reported radiographic findings of central sulcus narrowing. ${ }^{1}$ There was also displacement of brain tissue along the ventricular margins, consistent with enlargement of the ventricular system. No significant local changes in brain boundary were observed in the Shuttle astronauts following short-duration spaceflight.

\section{Associations between Structural Brain Changes and Motor Function}

On-line Table 2 shows the descriptive statistics (corrected for multiple comparisons) for the pre- and postflight scores for each of the functional task-testing domains, including the pronounced change in postural control as measured by sway speed on the Recovery from Fall/Stand Test and equilibrium score on the Dynamic Postural Stability Test.

To test for any voxelwise associations between brain volume change and motor function, we obtained nonparametric correlations between the normalized Jacobian determinant and each motor test score as a covariate. Local changes in brain structure were assessed using the Jacobian determinant, and several areas emerged as significant predictors of altered motor performance on postflight testing $(P<.05, n=8$, permutation-corrected) (Fig 3). Local structural change of the left caudate nucleus predicted a decrement in postural control as measured by the Recovery from Fall/Stand Test and the Dynamic Postural Stability Test. Prolonged completion time on the Seated Egress and Walk Test was predicted by local structural change of the right lower extremity primary motor area/midcingulate. No other brain areas demonstrated local changes in structure that correlated significantly with changes in postflight performance on the other FTT subtests.

We also tested for any association between $\% \Delta \mathrm{VV}$ and changes in motor test scores (On-line Table 3). There was a large correlation between $\% \Delta \mathrm{VV}$ and the sway speed on the Recovery from Fall/Stand Test $(r=0.74, n=8)$, indicating that those astronauts with the largest increases in $\% \Delta \mathrm{VV}$ had a greater loss of postural control postflight; however, this correlation did not survive correction for multiple comparisons.

\section{Associations between Structural Brain Changes and Cognitive Function}

Because of various mission lengths, individual astronauts had taken the WinSCAT between 9 and 12 times before the postflight examination; however, no significant association was seen between mission length and performance on any of the subtests of the WinSCAT. Given the small sample size and the repeated design at baseline, we did not attempt to correct for further potential learning effects among the astronauts.

On-line Table 4 shows the descriptive statistics for the preand postflight scores for each of the WinSCAT domains. Postflight, the astronauts demonstrated a significant (corrected for multiple comparisons) decrement in accuracy on the CDS 


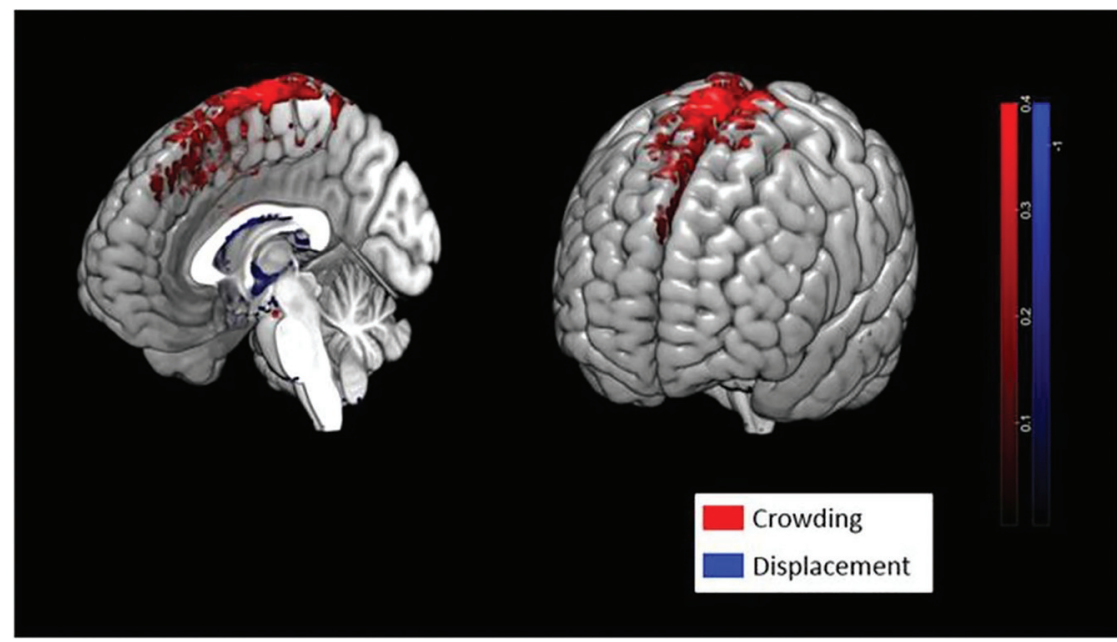

FIG 2. Spaceflight results in crowding of brain tissue at the vertex. Red voxels indicate regions along the brain surface where there was an increase in brain parenchyma pre- to postflight due to crowding of the brain tissue as the brain shifted upwards. Blue voxels indicate displaced brain tissue that occurred predominantly along the margins of the lateral and third ventricles due to enlargement of the ventricles postflight.

lumbar puncture following the spaceflight. Three additional astronauts demonstrated choroidal folds but did not undergo lumbar puncture. These 4 astronauts (all men) were classified as SANS cases. Compared with the 8 long-duration astronauts who did not develop findings of SANS, there was a smaller change in $\% \Delta \mathrm{VV}$ postflight in the 4 astronauts with SANS $(12.8 \%$ versus $6.5 \%, P=.02$ ). Because this was a post hoc comparison between 2 relatively small groups of people, we believe the findings should be considered preliminary rather than confirmatory.

\section{DISCUSSION}

Here we show that alteration of brain structure during spaceflight is likely progressive on the basis of mission duration, affects the younger astronauts, and correlates with postflight changes in motor and cognitive performance. These findings suggest that the brain

processing speed and learning subtest $(-0.8 \% \pm 1.1 \%, P=.02$, $n=12$ ) but faster reaction times for CDS and the CPT sustained attention subtest as indicated by negative percentage changes in reaction time $(-9.1 \% \pm 10.8 \%, P=.02$ and $-9.4 \% \pm 8.2 \%$, $P=.001$, respectively; $n=12$ ).

To test for any voxelwise associations between brain volume change and cognitive function, we obtained nonparametric correlations between the normalized Jacobian determinant and each cognitive test score as a covariate. Three white matter regions emerged as significant predictors of altered reaction time on the CPT (bilateral optic radiations and splenium of the corpus callosum; $P<.05, n=12$, permutation-corrected) (Fig 4). Those astronauts with the least reduction in reaction time postflight showed the greatest change in local volume in these 3 white matter regions. No area of the brain demonstrated local structural change that correlated significantly with postflight performance changes on any other WinSCAT subtests in this relatively small sample.

We also tested for any association between $\% \Delta \mathrm{VV}$ and changes in cognitive test scores (On-line Table 5). While there was a large negative correlation between accuracy on the CDS subtest and the $\% \Delta \mathrm{VV}(r=-0.60, n=12)$, indicating a decrement in accuracy in astronauts with the greatest increases in $\% \Delta$ $\mathrm{VV}$, and a large negative correlation between $\% \Delta \mathrm{VV}$ and reaction time on the CPT subtest ( $r=-0.62, n=12$ ), indicating that those astronauts with smaller increases in $\% \Delta \mathrm{VV}$ exhibited slower reaction times postflight, neither of these correlations survived correction for multiple comparisons.

\section{Associations between Structural Brain Changes and Clinical Findings of SANS}

Of the 12 long-duration astronauts, 1 astronaut had optic disc edema and increased intracranial pressure $\left(21.5 \mathrm{~cm} \mathrm{H}_{2} \mathrm{O}\right)$ at changes seen with microgravity have measurable behavioral consequences. While observed in a relatively small sample, with some variation based on astronaut demographics, these results may have major health significance that should be considered in spaceflight policy and planning.

The results suggest that long-duration spaceflight causes both global and local changes in brain structure. There is a redistribution of CSF, which occurs with ventricular enlargement, a global upward brain shift, and narrowing of the CSF spaces at the vertex along the inner table of the skull. ${ }^{1}$ As a result, significant local changes include crowding of brain parenchyma along the superior-medial aspects of the frontal and parietal lobes and displacement of brain parenchyma along the margins of the lateral and third ventricles as the ventricles expand (Fig 2).

For the 2 FTT tasks requiring the greatest demand for dynamic control of postural equilibrium, we found a significant association between altered postural control postflight and local structural changes of the left caudate nucleus, an important structure in postural and locomotion control. ${ }^{15-17}$ For example, the caudate nucleus has been proposed to play a role in the pathophysiology of idiopathic normal pressure hydrocephalus, in which patients commonly present with abnormal gait and enlarged ventricles on brain imaging and have been found to have reduced volume ${ }^{18}$ and hypometablism ${ }^{19}$ of the caudate nuclei compared with healthy controls. Restoration of normal perfusion of the caudate nuclei after shunt placement has been shown to correlate with improved gait in patients with idiopathic normal pressure hydrocephalus. ${ }^{20}$ Similarly, in astronauts, our current findings suggest that structural changes of the caudate nucleus may contribute to the substantial deficits in postural control experienced by astronauts postflight. ${ }^{10}$ We hypothesize that unilateral association with the left caudate nucleus is due to the 

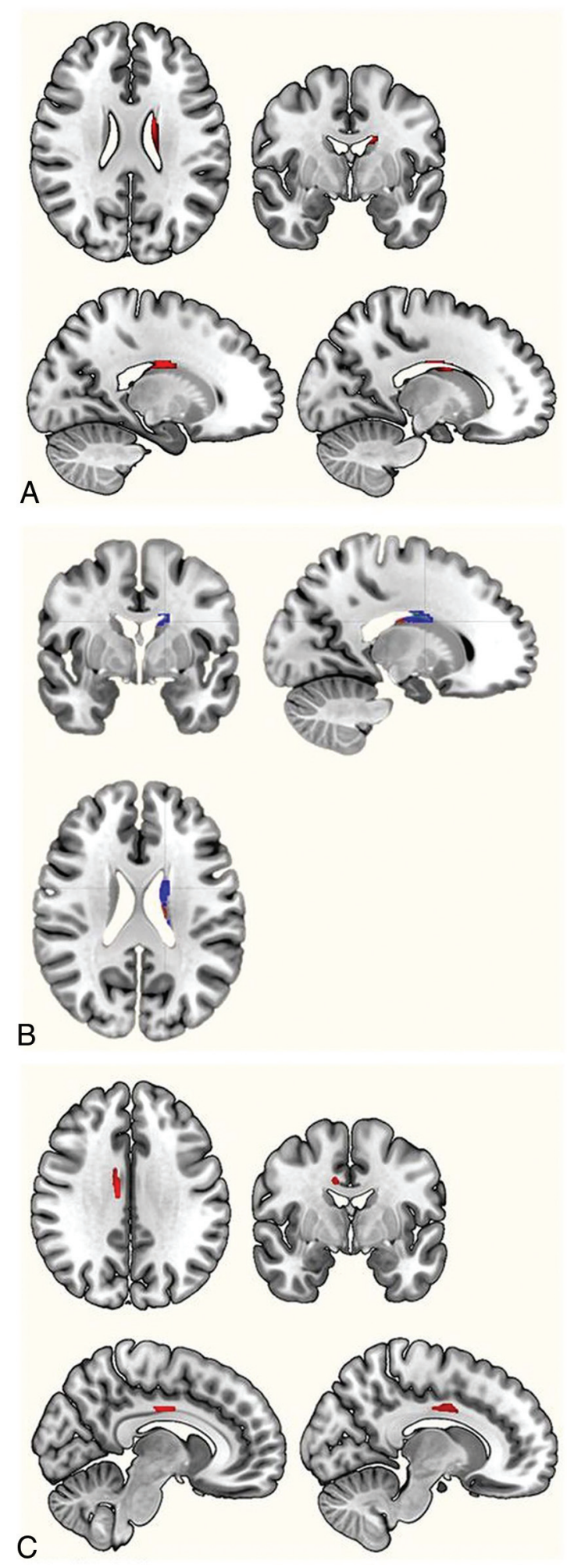

FIG 3. Regional deformation of brain parenchyma that significantly predicted performance on motor function tests. A, Regional deformation of brain parenchyma that significantly predicted performance on the Recovery from Fall/Stand Test $(P<.05)$. $B$, Regional deformation of brain parenchyma that significantly predicted performance on the Dynamic Postural Stability Test $(P<.05)$. $C$, Regional deformation of brain parenchyma that significantly predicted performance on the Seated Egress and Walk Test $(P<.05)$.
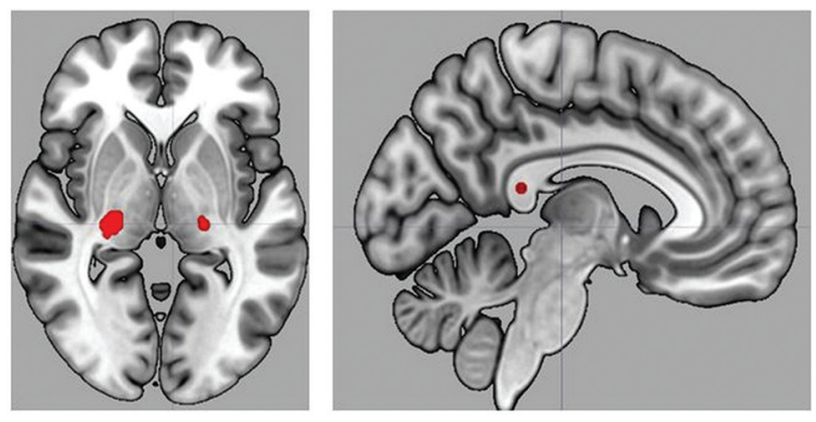

FIG 4. Regional deformation of brain parenchyma that significantly predicted performance on the Continuous Performance Test $(P<.05)$.

larger increase in left-versus-right ventricular volume (17.1\% versus $15.2 \%)$, resulting in a large change in the volume of the left caudate nucleus.

Alternatively, for the Seated Egress and Walk Test, we found a significant association between task-completion time and local structural changes of the right lower extremity primary motor area/midcingulate. The Seated Egress and Walk Test is a complex task that involves completing an obstacle course and therefore would be expected to engage higher order cortical areas. Unilateral association with the right motor area is consistent with previous results in which exposure to simulated microgravity caused a decrease in the activity of the left prefrontal and parietal cortices involved in preparation and realization of motor responses with a compensatory increase in right hemispheric activation. ${ }^{21}$ Pechenkova et $\mathrm{al}^{22}$ have also demonstrated several asymmetric alterations in motor system connectivity following spaceflight in a group of cosmonauts.

Postflight, the astronauts demonstrated reduced accuracy on the CDS subtest and faster reaction times for CDS and CPT. The change in reaction time could indicate ongoing learning effects during spaceflight on cognitive tasks as has been previously suggested. ${ }^{23}$ For example, Basner et $\mathrm{al}^{24}$ performed a normative study in astronauts, astronaut candidates, and NASA mission controllers using a test battery known as Cognition, which includes 2 subtests comparable with the CDS and CPT of the WinSCAT. Each participant performed the Cognition battery repeatedly to document the trajectory of speed and accuracy measures, and during 15 administrations of Cognition, the response times continued to decrease, suggesting ongoing learning. Alternatively, a change in reaction time and decreased accuracy on the CDS subtest may reflect a trade-off of accuracy for faster reaction times. A similar effect was also documented in the study of Basner et $\mathrm{al}^{24}$ for the CDS subtest. Faster reaction times were also seen at cognitive testing in a spaceflight analog study in which participants underwent long-term head-down-tilt bed rest simulating the headward fluid shifts of spaceflight while exposed to elevated carbon dioxide levels similar to those on the ISS. The authors suggested that the chronic exposure to elevated carbon dioxide experienced on the ISS may lead to altered cerebrovascular reactivity favoring the visual cortex and improved visual performance. ${ }^{25}$ Indeed, many factors affect individual astronaut performance, including hypercapnia, sleep, stress levels, noise, medications, and other psychosocial factors; therefore, further 
studies with larger sample sizes are needed to characterize cognitive performance in astronauts. Our current findings suggest individual brain structural adaption during spaceflight may also be an important factor affecting astronaut cognitive performance.

Local volume change of the right greater than left optic radiations and splenium of the corpus callosum was associated with relatively unaltered reaction times on the CPT compared with those astronauts who displayed faster reaction times on postflight testing. This finding is consistent with the known role of the splenium of the corpus callosum in mediating fast visuomotor responses. ${ }^{26}$ In astronauts, abnormal diffusion metrics involving multiple white matter tracts indicative of disrupted white matter connectivity have been identified postflight, ${ }^{27}$ and in particular, a recent study found focally reduced fractional anisotropy within the right posterior thalamic radiation in astronauts postflight, a physiologic correlate to the local volume change we identify here. $^{28}$

Initially, we had hypothesized that ventricular enlargement postflight would correlate with the development of SANS; however, the 4 astronauts with ophthalmologic changes had a smaller change in $\% \Delta \mathrm{VV}$ than those without SANS (12.8\% versus $6.5 \%$, $P=.02, n=8$ versus $n=4)$. While the etiology of SANS remains unclear, this preliminary result suggests that ventricular enlargement, as seen in astronauts without SANS, may not be a characteristic feature of SANS.

While there was a large correlation between $\% \Delta \mathrm{VV}$ and postflight sway speed (Recovery from Fall/Stand Test) and a large negative correlation with accuracy (CDS cognitive subtest), indicating that ventricular enlargement was associated with a loss of postflight postural control and a decrement in accuracy, these did not survive correction for multiple comparisons. There was also a large negative correlation between $\% \Delta \mathrm{VV}$ and reaction time on the CPT subtest, indicating that those astronauts with smaller increases in $\% \Delta \mathrm{VV}$ exhibited slower reaction times postflight. While this finding is consistent with the smaller change in $\% \Delta$ VV experienced by astronauts with SANS whose visual changes may have affected visuomotor performance, this finding also did not survive correction for multiple comparisons. Therefore, any association between $\% \Delta \mathrm{VV}$ and altered cognitive or motor performance will need to be studied in a larger group of astronauts.

The opportunity for individuals to experience spaceflight is limited; thus, the unique sample described here is important for guiding decision-making about future spaceflight. However, this uniqueness also limits the strength of inference that can be made from results involving a relatively small sample size, particularly for female astronauts who were under-represented in the current sample. Another limitation is the lack of uniform testing across all astronauts due to the retrospective nature of this study.

\section{CONCLUSIONS}

In the context of cautious interpretation, we found clear evidence of changes in brain structure that appear to occur with changes in behavior involving postural adjustment and reaction time during a cognitive task. The importance of these results is to highlight the need to prioritize further studies of human brain adaptation to spaceflight to ensure the safety of ISS astronauts and to prepare for long-duration exploration missions to the Moon and ultimately to Mars. Our findings support the need for advanced neuroimaging protocols and long-term follow-up imaging of the astronaut population. Most important, understanding the influences of gravity on CSF homeostasis and brain health may provide insights into abnormalities of CSF homeostasis such as idiopathic normal pressure hydrocephalus.

\section{ACKNOWLEDGMENTS}

We thank Emily Kreibich, Kaylen Bradley, and Brandon Dixon for assistance in data analysis. We thank Drs James Picano and Kimberly Seaton for assistance with interpretation of the WinSCAT data. We also thank Wafa Taiym and Sara Mason of the Lifetime Surveillance of Astronaut Health program and NASA Johnson Space Center, who provided the astronaut WinSCAT scores and imaging data. We thank members of the Functional Task Test study team at NASA Johnson Space Center for the FTT and balance data.

Disclosures: Donna R. Roberts-RELATED: Grant: National Aeronautics and Space Administration*; Support for Travel to Meetings for the Study or Other Purposes: National Aeronautics and Space Administration.* Truman R. BrownUNRELATED: Employment: Medical University of South Carolina. Paul J. NietertRELATED: Grant: National Institutes of Health, Comments: grant No. ULITR001450.* Dani Inglesby-RELATED: Grant: South Carolina Space Grant Minorities in STEM Student Scholarship. Mark S. George-RELATED: Grant: National Aeronautics and Space Administration.* Davud Asemani-RELATED: Grant: National Aeronautics and Space Administration. *Money paid to the institution.

\section{REFERENCES}

1. Roberts DR, Albrecht MH, Collins HR, et al. Effects of spaceflight on astronaut brain structure as indicated on MRI. $N$ Engl J Med 2017;377;1746-53 CrossRef Medline

2. Alperin N, Bagci AM, Lee SH. Spaceflight-induced changes in white matter hyperintensity burden in astronauts. Neurology 2017;89:2187-91 CrossRef Medline

3. Koppelmans V, Bloomberg JJ, Mulavara AP, et al. Brain structural plasticity with spaceflight. NPJ Microgravity 2016;2:2 CrossRef Medline

4. Van Ombergen A, Jillings S, Jeurissen B, et al. Brain tissue-volume changes in cosmonauts. $N$ Engl J Med 2018;379:1678-80 CrossRef Medline

5. Nicogossian AE, Huntoon CL, Pool SL. In: Nicogossian AE, Huntoon CL, Pool SL, eds.. Space Physiology and Medicine. 2nd ed. Philadelphia: Lea \& Febiger; 1989

6. Mader TH, Gibson CR, Pass AF, et al. Optic disc edema, globe flattening, choroidal folds, and hyperopic shifts observed in astronauts after long-duration space flight. Ophthalmology 2011;118:2058-69 CrossRef Medline

7. Lee AG, Mader TH, Gibson CR, et al. Space flight-associated neuroocular syndrome (SANS). Eye (Lond) 2018;32;1164-67 CrossRef Medline

8. Kane RL, Short P, Sipes W, et al. Development and validation of the spaceflight cognitive assessment tool for Windows (WinSCAT). Aviat Space Environ Med 2005;76(6 Suppl):B183-91 Medline

9. Seaton KA, Slack KJ, Sipes WA, et al. Cognitive functioning in longduration head-down bed rest. Aviat Space Environ Med 2009;80(5 Suppl);A62-65 CrossRef Medline

10. Mulavara AP, Peters BT, Miller CA, et al. Physiological and functional alterations after spaceflight and bed rest. Med Sci Sports Exerc 2018;50:1961-80 CrossRef Medline

11. Lee AG, Tarver WJ, Mader TH, et al. Neuro-ophthalmology of space flight. J Neuroophthalmol 2016;36:85-91 CrossRef Medline 
12. Winkler AM, Ridgway GR, Webster MA, et al. Permutation inference for the general linear model. Neuroimage 2014:92:381-97 CrossRef Medline

13. Cohen J. A power primer. Psychological Bulletin [PsycARTICLES] 1992;112:1 http://www.bwgriffin.com/workshop/Sampling\%20A\% 20Cohen\%20tables.pdf. Accessed May 22, 2019

14. Benjamini Y, Keiger AM, Yekutieli D. Adaptive linear step-up procedures that control the false discovery rate. Biometrika 2006;93:491-507 CrossRef

15. Takakusaki K, Oohinata-Sugimoto J, Saitoh K, et al. Role of basal ganglia-brainstem systems in the control of postural muscle tone and locomotion. Prog Brain Res 2004;143:231-37 CrossRef Medline

16. Takakusaki K, Tomita N, Yano M. Substrates for normal gait and pathophysiology of gait disturbances with respect to the basal ganglia dysfunction. J Neurol 2008;255(Suppl 4);19-29 CrossRef Medline

17. Villablanca JR. Why do we have a caudate nucleus? Acta Neurobiol Exp (Wars) 2010;70:95-105 Medline

18. DeVito EE, Salmond $\mathrm{CH}$, Owler BK, et al. Caudate structural abnormalities in idiopathic normal pressure hydrocephalus. Acta Neurol Scand 2007;1 16:328-32 CrossRef Medline

19. Townley RA, Botha H, Graff-Radford J, et al. (18)F-FDG PET-CT pattern in idiopathic normal pressure hydrocephalus. Neuroimage Clin 2018;18:897-902 CrossRef Medline

20. Ziegelitz D, Arvidsson J, Hellström P, et al. In patients with idiopathic normal pressure hydrocephalus postoperative cerebral perfusion changes measured by dynamic susceptibility contrast magnetic resonance imaging correlate with clinical improvement. J Comput Assist Tomogr 2015;39:531-40 CrossRef Medline

21. Kirenskaya AV, Tomilovskaya ES, Novototskii-Vlasov VY, et al. The effects of simulated microgravity on characteristics of slow presaccadic potentials [in Russian]. Fiziol Cheloveka 2006;32:131-39 Medline

22. Pechenkova E, Nosikova I, Rumshiskaya A, et al. Alterations of functional brain connectivity after long-duration spaceflight as revealed by fMRI. Front Physiol 2019;10:761 CrossRef Medline

23. Strangman GE, Sipes W, Beven G. Human cognitive performance in spaceflight and analogue environments. Aviat Space Environ Med 2014;85:1033-48 CrossRef Medline

24. Basner M, Savitt A, Moore TM, et al. Development and validation of the cognition test battery for spaceflight. Aerosp Med Hum Perform 2015;86:942-52 CrossRef Medline

25. Lee J, De Dios YE, Gadd NE,et al. The effects of 30 days of bed rest with elevated carbon dioxide on cognitive performance. In: Proceedings of the Annual Meeting of the Society for Neuroscience, San Diego, California. November 3-7, 2018

26. Peru A, Beltramello A, Moro V, et al. Temporary and permanent signs of interhemispheric disconnection after traumatic brain injury. Neuropsychologia 2003;41:634-43 CrossRef Medline

27. Lee JK, Koppelmans V, Riascos RF, et al. Spaceflight-associated brain white matter microstructural changes and intracranial fluid redistribution. JAMA Neurol 2019;76:412-19 CrossRef Medline

28. Riascos RF, Kamali A, Hakimelahi R, et al. Longitudinal analysis of quantitative brain MRI in astronauts following microgravity exposure. J Neuroimaging 2019;29:323-30 CrossRef Medline 